EPJ Web of Conferences 64, 05005 (2014)

DOI: $10.1051 /$ epjconf/ 20146405005

(C) Owned by the authors, published by EDP Sciences, 2014

\title{
Magnetospheric outflows in young stellar objects
}

\author{
Claudio Zanni ${ }^{1, a}$ \\ ${ }^{1}$ INAF - Osservatorio Astrofisico di Torino, Strada Osservatorio 20, 10025, Pino Torinese, Italy
}

\begin{abstract}
Different classes of outflows are associated with the magnetospheric activity of accreting T Tauri protostars. Stellar winds are accelerated along the open field lines anchored in the stellar surface; disk winds (extended or X-type) can be launched along the open magnetic surfaces threading the accretion disk; another type of ejection can arise from the region of interaction of the closed magnetosphere with the accretion disk (magnetospheric ejections, conical winds), where the magnetic surfaces undergo quasiperiodic episodes of inflation and reconnection. In this chapter I will present the main dynamical properties of these different types of outflow. Two main issues will be addressed. First, I will try to understand if these ejection phenomena can account for the origin of the jets often observed in young forming stellar systems. Second, I will evaluate the impact of these outflows on the angular momentum evolution of the central protostar.
\end{abstract}

\section{Introduction}

Classical T Tauri stars (CCTS) are magnetically active pre-main sequence stars that often display supersonic outflows up to scales of a few $100 \mathrm{AU}[1,2]$, whose presence is clearly correlated with accretion signatures from a circumstellar disk $[3,4]$. Their mass loss rate corresponds to $1-10 \%$ of the accretion rate [5] and they reach a terminal speed of the order of a few times the escape velocity of the protostar, with high-velocity components $\left(200-400 \mathrm{Km} \mathrm{s}^{-1}\right)$ coexisting with low-velocity ones $\left(<100 \mathrm{Km} \mathrm{s}^{-1}\right)$ [6]. The narrow opening angles of a few degrees observed beyond a few tens of AU from the source indicate that the jets must be efficiently collimated [2].

Despite a general consensus about the fact that magnetohydrodynamic (MHD) processes are the most viable mechanism to accelerate and self-collimate these jets on the spatial scales required by subarcsecond observations, the location of the launching site is still a matter of debate: are the outflows launched from the accretion disk, the stellar surface, the star-disk magnetospheric interaction region or a combination of the aforementioned? Since outflows coming from the magnetospheric region typically possess a lower specific angular momentum than winds coming farther from the disk, it has been proposed that measures of the jet rotation speed $[7,8]$ could be used to discern the launching site [9], but these measurements are rather controversial.

CTTS are characterized by strong surface magnetic fields up to a few kG [10], whose large-scale topology [11-13] allows to truncate the disk at a few stellar radii and channel the accretion flux into funnels flows that terminate with a shock at the stellar surface [14]. These magnetospheric fields are also strong enough to drive different types of outflows emerging from distinct parts of the innermost

ae-mail: zanni@oato.inaf.it 


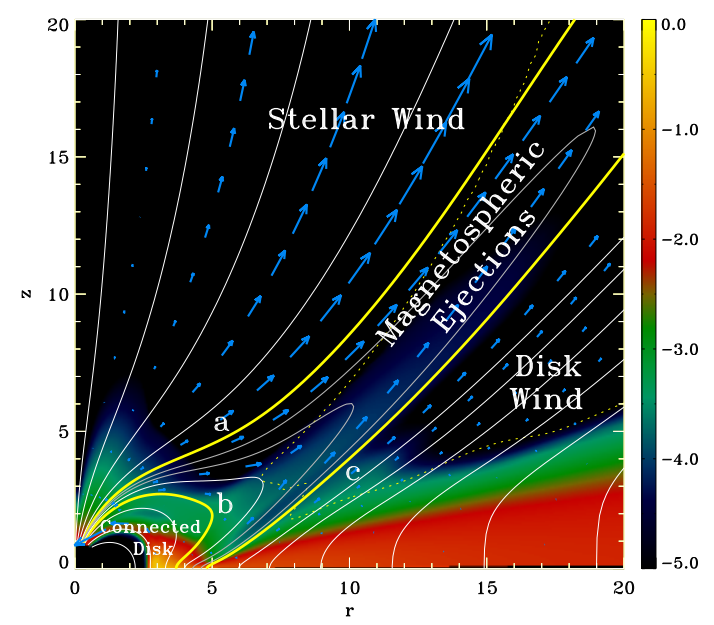

Figure 1. Global view of the outflows associated with the magnetospheric activity of a star-disk interacting system. Superposed to a logarithmic density map are sample magnetic field lines (solid lines), velocity vectors (blue arrows) and the Alfvén surface (dotted line). The thicker field lines, labeled as (a), (b) and (c) separate different dynamical constituents of the system. Taken from [22].

regions of the star-disk system (Fig. 1): the dynamical properties of these magnetospheric outflows (mass outflow rate, propagation speed, collimation degree, rotation signatures) can be compared with the observed jet kinematics.

In recent years, different studies about magnetospheric outflows have specifically addressed the problem of the evolution of the stellar angular momentum. As soon as they become visible after the Class 0-I embedded phases, a relevant fraction of CTTS appears to rotate well below their breakup limit, with rotation periods around 1-10 days [15]. Besides, their rotation period appears to be rather constant during the accreting evolutionary phases that last a few million years [16]. On the other hand, these protostars are still actively accreting and contracting so that they would be expected to spin-up at breakup in $\approx 10^{6}$ years. Clearly, CTTS require an efficient spin-down mechanism to explain their rotational evolution. Recently, the classical "disk-locking" paradigm proposed by Ghosh \& Lamb [17], based on a magnetically-driven angular momentum exchange between the star and the disk, has been critically re-examined: it has been realized that, (1) the size of the region steadily connecting the star with the disk is limited by the twisting of the field lines [18] and (2) the competition between accretion and diffusion is likely to reduce the magnetic field intensity in the region beyond the corotation radius and the corresponding spin-down torque [19]. As a consequence, different authors have reconsidered and further developed scenarios based on the presence of outflows that could efficiently extract angular momentum from the star-disk system [20-22].

In this chapter I will present the outcome of various semi-analytical and numerical models of the magnetospheric star-disk interaction aimed at modeling the main dynamical properties of different classes of magnetospheric outflows. I will compare them to the observed kinematic signatures of jets from CTTS and evaluate their contribution to the stellar angular momentum problem.

\section{Magnetospheric outflows}

An example of the typical outcome of a numerical simulation of the interaction of a dipolar stellar magnetosphere with a circumstellar accretion disk is depicted in Fig. 1. Notice that in the following I will take into account scenarios that exploit the dipolar component of the stellar magnetosphere only, while observations clearly indicate multipolar topologies [13,23]. Even if this component is seldom the strongest one, it is expected to control the dynamics on the scale of the disk-magnetosphere inter- 

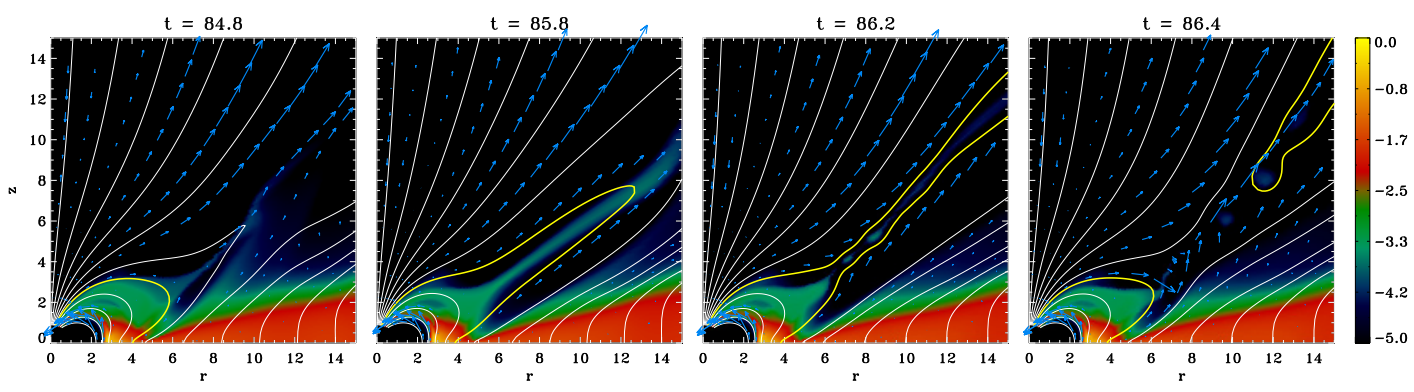

Figure 2. Temporal evolution of the expansion/reconnection process associated with magnetospheric ejections. Magnetic field lines (solid lines) and velocity vectors (blue lines) are superposed to logarithmic density maps. The thicker yellow solid line follows the evolution of a single magnetic surface. Time is in units of the stellar rotation period. Taken from [22].

action and beyond. Due to the differential rotation between the star and the disk and the consequent buildup of the toroidal field pressure, the initial closed dipolar structure relaxes by opening up. The resulting magnetic topology defines four regions, each associated with a different dynamical process: (1) a region of closed flux ("connected disk"), where the star and the disk can directly exchange angular momentum, the disk is truncated and the accretion curtains form; (2) an area of open flux at high stellar latitudes, where stellar winds can be accelerated; (3) the part of the disk threaded by open field lines, where disk winds can be launched; (4) a region of magnetic flux connecting the disk with the star, periodically evolving through stages of inflation, reconnection and contraction. An example of this periodic process is represented in Fig. 2. I will refer to the outflows associated with this last mechanism as magnetospheric ejections (MEs). The following subsections will be devoted to the analysis of the main dynamical properties of these three classes of magnetospheric outflows.

\subsection{Stellar winds}

The angular velocity of CTTS corresponds to a small fraction of their breakup speed, typically less than $10 \%$. As a consequence, magneto-centrifugal effects can not provide the initial drive to a wind at the stellar surface, while they are responsible for the large-scale acceleration and collimation. It has been proposed that the energy deposited by accretion at the stellar surface could provide an additional energy input (accretion-powered stellar winds, APSW, [20]). Actually, the broad blue-shifted absorptions in high excitation lines often observed in CTTS $[24,25]$ are usually interpreted as evidence for accretion-related stellar winds.

Therefore, the nature of the wind driving power remains controversial. Stellar winds are unlikely to be thermally driven: it has been shown that the temperature required to push such a flow $\left(\approx 10^{6}\right.$ $\mathrm{K}$ ) would substantially suppress the HeI and $\mathrm{H}$ spectral features usually associated with stellar winds while, for mass loss rates typically larger than $\approx 10^{-11} M_{\odot} \mathrm{yr}^{-1}$, the total wind luminosity would exceed the accretion power and produce too strong X-ray emission [26]. Turbulent Alfvén waves generated by the impact of the accretion streams onto the surface of the star could represent another possible pressure source $[27,28]$. In this case, the accretion/ejection coupling is not easy to determine: it has been suggested that the wind mass loss rates due to this mechanism are generally low, corresponding to less than $1 \%$ of the mass accretion rate [28].

On the other hand, it has been been shown that even stellar winds with a low mass loss rate are characterized by large magnetic lever arms [19]: stellar winds can reach high terminal speeds 
and extract a relevant fraction of the stellar angular momentum. It is possible to speculate that stellar winds likely represent an inner fast component of the observed jets. These characteristics have pushed to consider APSWs as a possible solution to the angular momentum problem [20]. A scaling relation between the torque exerted by the wind $\left(\tau_{\mathrm{w}}=\dot{M}_{\mathrm{sw}} R_{\mathrm{A}}^{2} \Omega_{\star}\right)$ and the main stellar parameters has been derived by $[29,30]$ :

$$
\tau_{\mathrm{w}}=K\left(2 G M_{\star}\right)^{-m} R_{\star}^{5 m-2} \dot{M}_{\mathrm{sw}}^{1-2 m} B_{\star}^{4 m} \Omega_{\star},
$$

where $M_{\star}$ is the stellar mass, $R_{\star}$ is its radius, $\Omega_{\star}$ is its angular rotation speed, $B_{\star}$ the dipolar field intensity at the stellar equator, $\dot{M}_{\mathrm{sw}}$ the wind mass flux and $R_{\mathrm{A}}$ is the wind magnetic lever arm, corresponding to the (average) cylindrical radius of the Alfvén surface. An estimate for the $m$ and $K$ coefficients based on a large set of MHD simulations of stellar winds (Fig. 3, left panel) has been provided by [30]:

$$
m \approx 2.1177 \quad K \approx 6.2\left[1+(\delta / 0.0716)^{2}\right]^{-m},
$$

where $\delta=\Omega_{\star} / \sqrt{G M_{\star} / R_{\star}^{3}}$ expresses the stellar rotation rate as a fraction of the breakup speed. The stellar wind spin-down torque defined by Eq. (1) and (2) reveals that a mass loss rate of the order of $\approx 10 \%$ of the accretion rate is needed to balance at least the spin-up torque due to accretion (see section 3). If the stellar contraction is taken into account, even heavier winds are required. Such winds would carry the entire mass flux typically observed in T Tauri jets, which seems both theoretically and observationally unlikely [5,9]. It has also been shown that, since the accretion energy must be used to power both the stellar wind and the observed accretion diagnostics (e.g., the UV excess luminosity, [14]), the observed accretion shock luminosity imposes even higher limits on the minimum mass loss rate and in some cases there is clearly not enough energy to power both the accretion shock emission and a stellar wind capable of balancing at least the accretion torque [31]. On the other hand, it has been shown that the stellar wind torque parametrized by Eq. (1) and (2) can account for the spin evolution of solar-like protostars after the end of the accreting phase up to the ZAMS.

\subsection{Disk winds}

As Fig. 1 shows, the opening of the dipolar magnetosphere provides open flux to the circumstellar disk, along which a disk wind can possibly be launched. Classical disk wind theory [34] states that a magnetic field close to equipartition with the disk thermal energy is needed to launch a powerful outflow. The observed magnetospheres can not provide enough flux to the disk to fulfill the equipartition condition over a large area of the disk extending well beyond the truncation/corotation radii. As a consequence, the extended disk winds observed in numerical simulations that include the stellar field only are not very strong, extracting at most a few percent of the accretion rate, and their Alfvén surface lies very close to the disk surface [22]. These light breezes can not reach a high terminal propagation speed and they exert only a weak torque on the disk. Therefore, their dynamical characteristics are not consistent with the observed jets and they play a negligible role on the evolution of the stellar rotation. Extended disk winds [34] therefore need a proper disk field to be powered (see J. Staff's contribution). Their characteristics seem to reproduce several features of the observed jets [5] so that they still represent one of the best candidates to explain the jet phenomenon in CTTS. A scenario in which a disk field interacts with the stellar magnetic flux has been proposed (Reconnection X-wind, [35]) and it deserves future investigation.

On the other hand, if the open stellar flux is compressed in a small region of the disk, the local magnetic field becomes strong enough to potentially launch a more energetic outflow. This idea is exploited by the $\mathrm{X}$-wind scenario [36], where the launching region of a fan-shaped wind and the truncation radius of the disk are located in a tiny region with a radial extent $\Delta r \approx h$ (where $h$ is the 

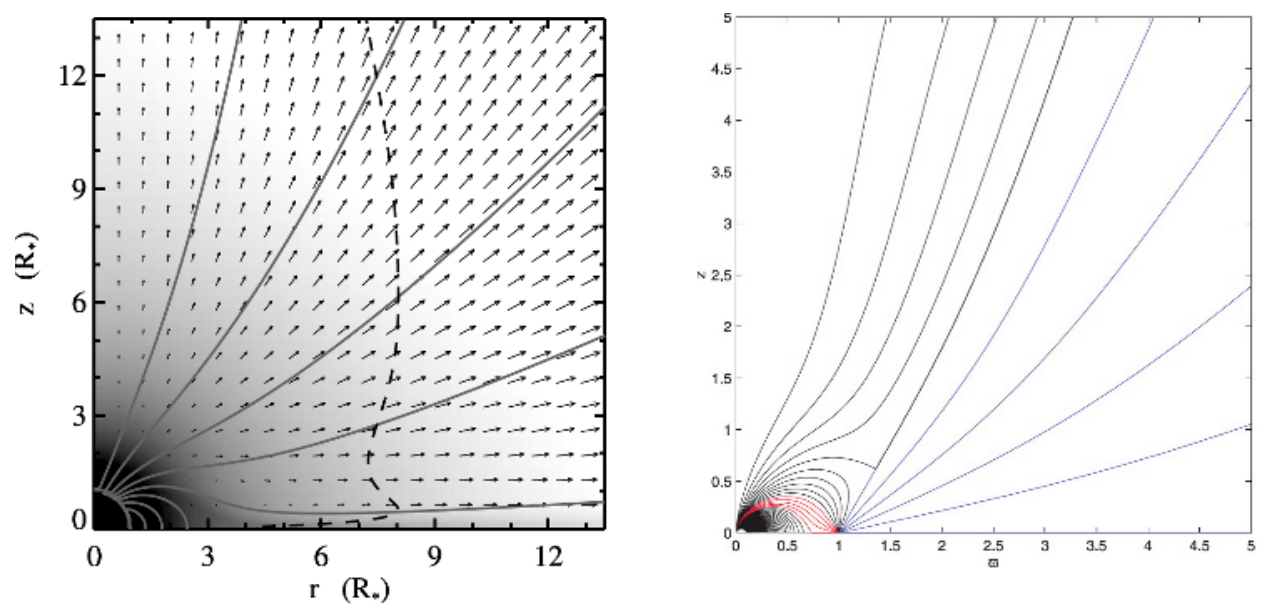

Figure 3. Left panel. Example of a simulation of an isolated stellar wind (i.e. without the presence of a circumstellar disk) employed by $[29,30]$ to parametrize the stellar wind torque. Magnetic field lines (solid lines), velocity vectors (arrows) and the Alfvén surface (dashed line) are pictured. Right panel. Streamlines and magnetic field lines of an $\mathrm{X}$-wind (blue lines, [32]). The funnel flow (red lines) and the regions that the $\mathrm{X}$-wind scenario considers as dead to magnetocentrifugal fling (black lines) are taken from [33].

disk half-thickness) around the corotation radius $R_{\mathrm{co}}=\left(G M_{\star} / \Omega_{\star}^{2}\right)^{1 / 3}$, which represents a saddle ("X" point) of the gravito-centrifugal potential. Consistently with the values inferred from observations, the model imposes a ratio between mass loss and accretion rates $f=\dot{M}_{\mathrm{xw}} / \dot{M}_{\mathrm{acc}} \approx 0.1-0.3$ and a magnetic lever arm of the order $R_{\mathrm{A}} \approx R_{\mathrm{co}} / \sqrt{f}$. This value of the magnetic lever arm provides an appropriate terminal speed $\left(v_{\infty}^{2} \approx\left[2\left(R_{\mathrm{A}} / R_{\mathrm{co}}\right)^{2}-3\right] G M_{\star} / R_{\mathrm{co}}\right)$, even if the asymptotic structure of the jet does not show a large velocity spread [5], as often observed in T Tauri jets. Correspondingly, the torque onto the disk $\left(\dot{M}_{\mathrm{xw}} R_{\mathrm{A}}^{2} \Omega_{\star}=\dot{M}_{\mathrm{xw}} \sqrt{G M_{\star} R_{\mathrm{co}}} / f=\dot{M}_{\text {acc }} \sqrt{G M_{\star} R_{\mathrm{co}}}\right)$ can extract all the angular momentum accreted by the disk at corotation, so as to cancel the accretion spin-up torque [33].

Models of trans-Alfvénic fan-shaped outflows with characteristics similar to what prescribed by the X-wind model can be computed ([32,37], Fig. 3, right panel). These models, both semi-analytical and numerical, use fixed boundary conditions at the launching site (e.g. rotation, mass and magnetic flux distribution) but neglect the actual dynamical connection with the disk, that represents the most crucial issue of the $\mathrm{X}$-wind scenario. In order to compress all the open flux in a small region around corotation ("flux-trapping", in the X-wind jargon), the X-wind magnetic configuration requires a magnetic Prandtl number, given by the ratio between the disk (turbulent) viscosity and magnetic diffusivity, of order $r / h \gg 1$. Our current knowledge about MRI-driven turbulence in accretion disks seems to favor magnetic Prandtl number values around unity [38]. Over such a small radial extent, the accretion disk does not contain enough mechanical energy to fuel an outflow with the typical characteristics of an X-wind, but it can launch a less massive/powerful outflow only. An X-wind requires an extra energy input: if we presume that this comes from the viscous transport of rotational energy coming below $R_{\text {co }}$, the corresponding transport coefficients assume rather extreme and maybe unphysical values [39]. Even the mass loss rate of the X-wind remains a free parameter of the model, while its value should come from a computation of the disk vertical equilibrium that is currently missing. Global numerical simulations show ejections coming from the magnetospheric interaction region but, 
while they share some similarities with the X-wind scenario, they still present some major differences, as it is discussed in the next subsection.

\subsection{Magnetospheric ejections}

MEs are expected to arise because of the expansion and subsequent reconnection of the closed magnetospheric field lines ([22], Fig. 2). The inflation process is a result of the star-disk differential rotation and the consequent build-up of toroidal magnetic field pressure. This is the same phenomenon that bounds the size of the magnetosphere connecting the star with the disk and limits the efficiency of the Ghosh \& Lamb mechanism. In principle, MEs can exchange mass, energy and angular momentum both with the disk and the star, since initially they are launched along magnetic field lines which still connect the star with the disk. On a relatively large spatial scale $\left(\approx 10-20 R_{\star}\right)$ they detach from the magnetosphere in a reconnection event and continue their propagation as magnetic islands disconnected from the central part of the star-disk system, in between the open magnetic surfaces anchored into the star and those anchored into the disk. Because of magnetic reconnection, the inner magnetic surfaces close again and the process repeats periodically.

Even if MEs can receive mass both from the disk and the star, numerical simulations show that most of the mass is provided by the disk, roughly around $5-20 \%$ of the mass accretion rate [22]. While the same energetic problems that affect stellar winds limit the mass load coming from the star, an enhanced vertical thermal pressure gradient due to the push of the accretion flow against the magnetospheric wall allows to extract a considerable amount of mass from a relatively small area of the disk, as in the case of X-winds. Contrary to X-winds, MEs extract considerably less angular momentum and energy from the disk. For example, while X-winds are assumed to extract all the disk angular momentum before it is accreted, so that the disk exerts a null torque onto the star, it has been estimated that MEs are able to reduce the accretion torque by a $30-50 \%$ factor only [22]. The accretion disk has enough mechanical energy to power the less energetic MEs without any extra input as in the case of $\mathrm{X}$-winds.

As long as they are still magnetically connected to the star, MEs are able to exchange energy and angular momentum directly with it. The direction of this exchange is mainly controlled by the differential rotation between the star and the MEs: if the ejected plasma rotates slower than the star, the MEs can extract energy and angular momentum directly from the star. In such a situation, MEs are powered both by the stellar and the disk rotation, as in a huge magnetic slingshot. Because of the torque exerted by the MEs, the disk and the MEs rotation becomes sub-Keplerian and, in some cases, even substellar below $R_{\mathrm{co}}$. As a consequence, MEs start to exert a spin-down torque onto the star even when the disk is truncated inside $R_{\mathrm{co}}$. According to the simulations presented by [22], it is possible to nullify the torque onto the stellar surface when the disk is truncated at a radius $R_{\mathrm{t}} \approx 0.6 R_{\mathrm{co}}$ already.

Obviously a zero-torque condition, when the spin-down mechanisms exactly balance the accretion spin-up torque, is not sufficient to keep the stellar rotation period constant, as observations seem to suggest. Since CTTS are still contracting, it is necessary to provide a braking torque so that the spindown timescale becomes comparable to the Kelvin-Helmholtz contraction timescale, approximately of the order of a few $10^{6}$ years. It has been found by [22] that this condition can be satisfied when the truncation further approaches the corotation radius and the system enters a propeller regime [40, 41]: in this situation, while the MEs dynamics are rather similar, the accretion rate becomes intermittent on a timescale corresponding to a few rotation periods of the star. Even though this effect can be enhanced by the axial symmetry of the models, there is as yet no observational evidence for such a behavior.

On a larger spatial scale, the MEs disconnect from the central region of the disk-star system in a magnetic reconnection event and propagate ballistically as magnetized plasmoids. Consistently, MEs 
can not be self-collimated thanks to magnetic stresses, as usually envisaged for magnetically-driven outflows. This confers the characteristic "conical" shape to these magnetospheric outflows [42]. This is clearly not compatible with the collimation observed in $\mathrm{T}$ Tauri jets. The collimation properties of MEs depend on the equilibrium between the inner stellar wind and an outer disk wind, among which MEs propagate. For example, it has been shown in [43] that, at least for high mass accretion rates, more typical of EXORs and FUORs, the innermost opened disk field lines can be sufficiently mass loaded so that the corresponding disk wind can confine the inner outflows. Besides, since MEs are rather massive, typically the energy that they extract from the star-disk system is not sufficient to accelerate them beyond $\approx 100 \mathrm{Km} \mathrm{s}^{-1}$, so that they could represent only a medium/low velocity component of the observed jets.

\section{Spin-down efficiency}

In this section, I am going to compare the spin-down efficiency of the outflow models presented in the previous sections by analyzing the spin-equilibrium condition predicted by each scenario. Most star-disk interaction models express the balance between spin-down and spin-up torques in terms of the relative position of the truncation radius with respect to the corotation radius. Since the Alfvén radius for spherical free-fall collapse onto a dipolar magnetosphere [44]:

$$
R_{\mathrm{A}, \mathrm{ff}}=\left(\frac{B_{\star}^{4} R_{\star}^{12}}{G M_{\star} \dot{M}_{\mathrm{acc}}^{2}}\right)^{1 / 7}
$$

has been shown to be a good proxy for the disk truncation radius, with typically $R_{\mathrm{t}}=0.5-1 R_{\mathrm{A}, \mathrm{ff}}$ $[19,45]$, the stellar rotation period corresponding to a zero-torque situation can be expressed as

$$
P_{\mathrm{eq}} \approx 10 K\left(\frac{B_{\star}}{1 \mathrm{kG}}\right)^{6 / 7}\left(\frac{R_{\star}}{2 R_{\odot}}\right)^{18 / 7}\left(\frac{M_{\star}}{0.5 M_{\odot}}\right)^{-5 / 7}\left(\frac{\dot{M}_{\mathrm{acc}}}{10^{-8} M_{\odot} \mathrm{yr}^{-1}}\right)^{-3 / 7} \text { days },
$$

where $K=\left(R_{\mathrm{co}} / R_{\mathrm{A}, \mathrm{ff}}\right)^{3 / 2}$. Clearly, a higher $K$ factor corresponds to a more efficient spin-down mechanism. For example, the X-wind model employs a value $K=0.89$ [33], while the MEs are slightly less efficient, with $K=0.54$ [22]. The application of the Ghosh \& Lamb model to CTTS has provided a value $K=0.87$ [46]. On the other hand, the corrections brought to this scenario by [18] provide an upper limit $K<0.3$ that, according to [19], is likely to be even lower.

The APSW model does not describe the spin-equilibrium in terms of the position of the truncation radius. By equating the torque expressed by Eq. (1) and (2) (with $\delta \leq 0.1$ ) with the accretion torque $\left(\tau_{\text {acc }}=\dot{M}_{\text {acc }} \sqrt{G M_{\star} R_{\mathrm{t}}} \approx \dot{M}_{\text {acc }} \sqrt{G M_{\star} R_{\mathrm{A}, \mathrm{ff}}}\right)$, the equilibrium period is given by:

$$
P_{\mathrm{eq}} \approx 6\left(\frac{\dot{M}_{\mathrm{sw}} / \dot{M}_{\mathrm{acc}}}{0.1}\right)^{0.55}\left(\frac{B_{\star}}{1 \mathrm{kG}}\right)^{0.61}\left(\frac{R_{\star}}{2 R_{\odot}}\right)^{2.26}\left(\frac{M_{\star}}{0.5 M_{\odot}}\right)^{-0.65}\left(\frac{\dot{M}_{\mathrm{acc}}}{10^{-8} M_{\odot} \mathrm{yr}^{-1}}\right)^{-0.3} \text { days } .
$$

Notice that the exponents are not very different from those of Eq. (4), while the $K$ factor has been replaced by a function of the ejection efficiency $\dot{M}_{\text {sw }} / \dot{M}_{\text {acc }}$. This equation clearly shows that an ejection efficiency $\approx 10 \%$ is necessary to give a characteristic rotation period comparable to the other scenarios.

Notice that Eq. (5) and (4) are meant to describe a null-torque condition, which is not a complete solution of the angular momentum problem of CTTS, since the stellar contraction is not taken into account. In order to counteract the stellar contraction to keep a roughly constant rotation period, an even stronger spin-down torque is necessary. In the case of APSW and X-winds this condition 
requires even more powerful outflows, while sections 2.1 and 2.2 showed that their power source is one of the main issues of both scenarios. MEs can in principle balance the spin-up due to contraction during a propeller regime, but this phase is characterized by an extreme accretion variability, which is currently not observed. Finally, all the presented scenarios need a $\mathrm{kG}$ dipolar component to exert an efficient enough torque, both in a spin-equilibrium situation, as shown by Eq. (4) and (5), and when the stellar contraction is considered.

\section{Summary}

In this chapter I have presented the main dynamical properties of different types of magnetospheric outflows (stellar winds, disk winds and magnetospheric ejections) in order to compare them with the kinematics inferred from observations of $\mathrm{T}$ Tauri jets and to evaluate their impact on the angular momentum evolution of the forming protostar.

- Stellar winds. Since CTTS are slow rotators and their centrifugal push is not strong enough to drive a stellar wind, an extra energy input is required. It has been proposed by [20] that this could come from the accretion power deposited at the stellar surface by the accreting material (accretionpowered stellar winds), but the functioning and efficiency of the accretion/ejection coupling remains uncertain. For example, it has been estimated by [28] that turbulent Alfvén waves excited by the impact of the accretion streams onto the stellar surface can yield a mass loss rate corresponding to around $1 \%$ of the accretion rate at most. These light winds can reach a high terminal speed and could represent a fast component in the inner layers of the observed jets. On the other hand, a stellar wind extracting at least $10 \%$ of the accretion rate is necessary to balance the accretion torque [47]. Such a mass loss rate is energetically very demanding and the situation becomes even more critical if the stellar contraction is taken into account [48].

- Disk winds. The observed magnetospheres can not provide enough open flux to the accretion disk to produce a relevant extended disk wind launched from a sizable area of the disk beyond the corotation radius. An extended disk wind requires a proper disk field distribution to be powered. If the stellar open flux is compressed in a small radial extent in the truncation/corotation region, a fanshaped X-wind can in principle be launched [36]. This scenario had been originally developed to account for the origin of $\mathrm{T}$ Tauri jets, with acceleration and collimation properties compatible with observations, and at the same time, to play a major role in the stellar spin evolution. The X-wind should in fact exert a torque so as to extract all the disk angular momentum before it is accreted, so that the accretion flow does not exert any torque onto the star. Even if this is the only spin-down model that has been extended to multipolar fields [21], the current formulation of the scenario does not take into account the stellar contraction. Besides, the dynamical connection of these outflows with the underlying disk has been criticized [39] and even support from numerical simulations has so far been controversial.

- Magnetospheric ejections. MEs arise because of the events of expansion and reconnection of the magnetic field lines connecting the star with the disk [22]. This process is a consequence of the buildup of toroidal field pressure due to the star-disk differential rotation. While the mass content of MEs is mainly provided by the disk, they can exchange energy and angular momentum both with the disk and the star, since they initially propagate along closed field lines. After a reconnection event, they disconnect and propagate ballistically as uncollimated plasmoids. MEs can not explain the jet phenomenon in $\mathrm{T}$ Tauri stars, since their terminal velocity is unlikely to be higher than the gravitational escape speed and they are poorly collimated. They have an important effect onto the stellar spin evolution. On one hand, they extract angular momentum from the disk close to the 
Physics at the Magnetospheric Boundary

truncation region so that the spin-up accretion torque is sensibly reduced, though less efficiently than in the X-wind scenario. On the other hand, they can extract angular momentum directly from the star thanks to a differential rotation effect. MEs start to balance the accretion torque even when the disk is truncated below the corotation radius and in a propeller phase they can even compensate the stellar contraction. Anyway, a few issues hold. The charge and discharge quasi-periodic process that characterizes MEs depends on magnetic reconnection events that in numerical models are controlled by the numerical dissipation only. The strong accretion variability that characterizes the propeller phases does not seem to be consistent with observations.

One limitation affects the efficiency of the spin-down torques of all the proposed models. In order to balance accretion and contraction, all the scenarios require a dipolar component of about $1-2 \mathrm{kG}$ ([48], F. Gallet in these proceedings). While this has been observed, for example, in the case of AA Tau [12], such a strong dipolar component does not seem to be a common feature among $\mathrm{T}$ Tauri stars $[11,13,23]$. While a larger sample of magnetic field measurements is clearly needed, torque models should consider more realistic magnetic field configurations, including non axisymmetric and multipolar components $[49,50]$.

\section{References}

[1] C.J. Burrows, K.R. Stapelfeldt, A.M. Watson, J.E. Krist, G.E. Ballester, J.T. Clarke, D. Crisp, J.S. Gallagher, III, R.E. Griffiths, J.J. Hester et al., Astrophys. J. 473, 437 (1996)

[2] C. Dougados, S. Cabrit, C. Lavalley, F. Ménard, Astron. \& Astrophys. 357, L61 (2000)

[3] S. Cabrit, S. Edwards, S.E. Strom, K.M. Strom, Astrophys. J. 354, 687 (1990)

[4] S. Edwards, W. Fischer, L. Hillenbrand, J. Kwan, Astrophys. J. 646, 319 (2006)

[5] S. Cabrit, The accretion-ejection connexion in T Tauri stars: jet models vs. observations, in IAU Symposium, edited by J. Bouvier, I. Appenzeller (2007), Vol. 243 of IAU Symposium, pp. 203-214

[6] F. Bacciotti, R. Mundt, T.P. Ray, J. Eislöffel, J. Solf, M. Camezind, Astrophys. J. Lett. 537, L49 (2000)

[7] F. Bacciotti, T.P. Ray, R. Mundt, J. Eislöffel, J. Solf, Astrophys. J. 576, 222 (2002)

[8] D. Coffey, F. Bacciotti, T.P. Ray, J. Eislöffel, J. Woitas, Astrophys. J. 663, 350 (2007)

[9] J. Ferreira, C. Dougados, S. Cabrit, Astron. \& Astrophys. 453, 785 (2006)

[10] C.M. Johns-Krull, Astrophys. J. 664, 975 (2007)

[11] G.A.J. Hussain, A. Collier Cameron, M.M. Jardine, N. Dunstone, J. Ramirez Velez, H.C. Stempels, J.F. Donati, M. Semel, G. Aulanier, T. Harries et al., Mon. Not. Roy. Astron. Soc. 398, 189 (2009)

[12] J.F. Donati, M.B. Skelly, J. Bouvier, S.G. Gregory, K.N. Grankin, M.M. Jardine, G.A.J. Hussain, F. Ménard, C. Dougados, Y. Unruh et al., Mon. Not. Roy. Astron. Soc. 409, 1347 (2010)

[13] J.F. Donati, S.G. Gregory, S.H.P. Alencar, J. Bouvier, G. Hussain, M. Skelly, C. Dougados, M.M. Jardine, F. Ménard, M.M. Romanova et al., Mon. Not. Roy. Astron. Soc. 417, 472 (2011)

[14] E. Gullbring, N. Calvet, J. Muzerolle, L. Hartmann, Astrophys. J. 544, 927 (2000)

[15] J. Bouvier, S. Cabrit, M. Fernandez, E.L. Martin, J.M. Matthews, Astron. \& Astrophys. 272, $176(1993)$

[16] J. Irwin, J. Bouvier, The rotational evolution of low-mass stars, in IAU Symposium, edited by E.E. Mamajek, D.R. Soderblom, R.F.G. Wyse (2009), Vol. 258 of IAU Symposium, pp. 363-374

[17] P. Ghosh, F.K. Lamb, Astrophys. J. 234, 296 (1979)

[18] S. Matt, R.E. Pudritz, Mon. Not. Roy. Astron. Soc. 356, 167 (2005a) 
[19] C. Zanni, J. Ferreira, Astron. \& Astrophys. 508, 1117 (2009)

[20] S. Matt, R.E. Pudritz, Astrophys. J. Lett. 632, L135 (2005)

[21] S. Mohanty, F.H. Shu, Astrophys. J. 687, 1323 (2008)

[22] C. Zanni, J. Ferreira, Astron. \& Astrophys. 550, A99 (2013)

[23] S.G. Gregory, J.F. Donati, J. Morin, G.A.J. Hussain, N.J. Mayne, L.A. Hillenbrand, M. Jardine, Astrophys. J. 755, 97 (2012)

[24] S. Edwards, W. Fischer, J. Kwan, L. Hillenbrand, A.K. Dupree, Astrophys. J. Lett. 599, L41 (2003)

[25] A.K. Dupree, N.S. Brickhouse, G.H. Smith, J. Strader, Astrophys. J. Lett. 625, L131 (2005)

[26] S. Matt, R.E. Pudritz, The nature of stellar winds in the star-disk interaction, in IAU Symposium, edited by J. Bouvier, I. Appenzeller (2007), Vol. 243 of IAU Symposium, pp. 299-306

[27] W.M. DeCampli, Astrophys. J. 244, 124 (1981)

[28] S.R. Cranmer, Astrophys. J. 706, 824 (2009)

[29] S. Matt, R.E. Pudritz, Astrophys. J. 678, 1109 (2008a)

[30] S.P. Matt, K.B. MacGregor, M.H. Pinsonneault, T.P. Greene, Astrophys. J. Lett. 754, L26 (2012a)

[31] C. Zanni, J. Ferreira, Astrophys. J. Lett. 727, L22 (2011)

[32] M.J. Cai, H. Shang, H.H. Lin, F.H. Shu, Astrophys. J. 672, 489 (2008)

[33] E.C. Ostriker, F.H. Shu, Astrophys. J. 447, 813 (1995)

[34] J. Ferreira, Astron. \& Astrophys. 319, 340 (1997)

[35] J. Ferreira, G. Pelletier, S. Appl, Mon. Not. Roy. Astron. Soc. 312, 387 (2000)

[36] F. Shu, J. Najita, E. Ostriker, F. Wilkin, S. Ruden, S. Lizano, Astrophys. J. 429, 781 (1994)

[37] J.M. Anderson, Z.Y. Li, R. Krasnopolsky, R.D. Blandford, Astrophys. J. 630, 945 (2005)

[38] G. Lesur, P.Y. Longaretti, Astron. \& Astrophys. 504, 309 (2009)

[39] J. Ferreira, F. Casse, Mon. Not. Roy. Astron. Soc. 428, 307 (2013)

[40] G.V. Ustyugova, A.V. Koldoba, M.M. Romanova, R.V.E. Lovelace, Astrophys. J. 646, 304 (2006)

[41] C.R. D’Angelo, H.C. Spruit, Mon. Not. Roy. Astron. Soc. 420, 416 (2012)

[42] M.M. Romanova, G.V. Ustyugova, A.V. Koldoba, R.V.E. Lovelace, Mon. Not. Roy. Astron. Soc. 399, 1802 (2009)

[43] P. Lii, M. Romanova, R. Lovelace, Mon. Not. Roy. Astron. Soc. 420, 2020 (2012)

[44] R.F. Elsner, F.K. Lamb, Astrophys. J. 215, 897 (1977)

[45] N. Bessolaz, C. Zanni, J. Ferreira, R. Keppens, J. Bouvier, Astron. \& Astrophys. 478, 155 (2008)

[46] A. Königl, Astrophys. J. Lett. 370, L39 (1991)

[47] S. Matt, R.E. Pudritz, Astrophys. J. 681, 391 (2008b)

[48] S.P. Matt, G. Pinzón, T.P. Greene, R.E. Pudritz, Astrophys. J. 745, 101 (2012b)

[49] M.M. Romanova, M. Long, F.K. Lamb, A.K. Kulkarni, J.F. Donati, Mon. Not. Roy. Astron. Soc. 411, 915 (2011)

[50] A.A. Vidotto, M. Jardine, M. Opher, J.F. Donati, T.I. Gombosi, Mon. Not. Roy. Astron. Soc. 412, $351(2011)$ 